\title{
Compact narrowband bandpass filter using dual-mode octagonal meandered loop resonator For WiMax application
}

\begin{abstract}
In this paper, a new design of a compact narrowband bandpass filter is proposed. This new narrowband bandpass filter is designed using an octagonal form of dual-mode closed-loop microstrip ring resonator based on a meander structure in order to achieve compactness. The designed filter has a $3 \mathrm{~dB}$ fractional bandwidth (FBW) of $5 \%$ at $2.3 \mathrm{GHz}$. The filter has been fabricated on Taconic CER-10 substrate having $0.64 \mathrm{~mm}$ thickness and a relative dielectric constant of 10. Experimental results show good agreement with simulated values. Apart from WiMax, this new model of filter is also useful for WLAN and mobile communication applications, since it is compact in size, low loss, and low cost with good performance of elliptic response with sharp rejection and adequate fractional bandwidth.
\end{abstract}

Keyword: Compact narrowband bandpass filter; Dual-mode; Octagonal meandered loop; WiMax 